\title{
Ideal-Gas Like Markets: Effect of Savings
}

\author{
Arnab Chatterjee and Bikas K Chakrabarti \\ Theoretical Condensed Matter Physics Division and Centre for Applied \\ Mathematics and Computational Science, Saha Institute of Nuclear Physics, \\ Block-AF, Sector-I Bidhannagar, Kolkata-700064, India. \\ arnab.chatterjee@saha.ac.in, bikask.chakrabarti@saha.ac.in
}

Summary. We discuss the ideal gas like models of a trading market. The effect of savings on the distribution have been thoroughly reviewed. The market with fixed saving factors leads to a Gamma-like distribution. In a market with quenched random saving factors for its agents we show that the steady state income $(m)$ distribution $P(m)$ in the model has a power law tail with Pareto index $\nu$ equal to unity. We also discuss the detailed numerical results on this model. We analyze the distribution of mutual money difference and also develop a master equation for the time development of $P(m)$. Precise solutions are then obtained in some special cases.

\section{Introduction}

The distribution of wealth among individuals in an economy has been an important area of research in economics, for more than a hundred years. Pareto [1] first quantified the high-end of the income distribution in a society and found it to follow a power-law

$$
P(m) \sim m^{-(1+\nu)}
$$

where $P$ gives the normalized number of people with income $m$, and the exponent $\nu$ is called the Pareto index.

Considerable investigations with real data during the last ten years revealed that the tail of the income distribution indeed follows the above mentioned behavior and the value of the Pareto index $\nu$ is generally seen to vary between 1 and 3 2, 3, 4, 5. It is also known that typically less than $10 \%$ of the population in any country possesses about $40 \%$ of the total wealth of that country and they follow the above law. The rest of the low income population, in fact the majority ( $90 \%$ or more), follow a different distribution which is debated to be either Gibbs [3, 6, 7] or log-normal [4.

Much work has been done recently on models of markets, where economic (trading) activity is analogous to some scattering process [6, 8, 9, 10, 11, 12 [13, 14, 15, 16] as in the kinetic theory [17] of gases or liquids. 
We put our attention to models where introducing a saving propensity (or factor) 18 for the agents, a wealth distribution similar to that in the real economy can be obtained [8, 12. Savings do play an important role in determining the nature of the wealth distribution in an economy and this has already been observed in some recent investigations [19]. Two variants of the model have been of recent interest; namely, where the agents have the same fixed saving factor [8], and where the agents have a quenched random distribution of saving factors [12]. While the former has been understood to a certain extent (see e.g, 20, 21]), and argued to resemble a gamma distribution 21. attempts to analyze the latter model are still incomplete (see however 22]). Further numerical studies [23] of time correlations in the model seem to indicate even more intriguing features of the model. In this paper, we intend to analyze the second market model with randomly distributed saving factor, using a master equation type approach similar to kinetic models of condensed matter.

We have studied here numerically a gas model of a trading market. We have considered the effect of saving propensity of the traders. The saving propensity is assumed to have a randomness. Our observations indicate that Gibbs and Pareto distributions fall in the same category and can appear naturally in the century-old and well-established kinetic theory of gas [17): Gibbs distribution for no saving and Pareto distribution for agents with quenched random saving propensity. Our model study also indicates the appearance of self-organized criticality [24] in the simplest model so far, namely in the kinetic theory of gas models, when the stability effect of savings [18] is incorporated.

\section{Ideal-gas like models}

We consider an ideal-gas model of a closed economic system where total money $M$ and total number of agents $N$ is fixed. No production or migration occurs and the only economic activity is confined to trading. Each agent $i$, individual or corporate, possess money $m_{i}(t)$ at time $t$. In any trading, a pair of traders $i$ and $j$ randomly exchange their money [6, 7, 8, such that their total money is (locally) conserved and none end up with negative money $\left(m_{i}(t) \geq 0\right.$, i.e, debt not allowed):

$$
m_{i}(t)+m_{j}(t)=m_{i}(t+1)+m_{j}(t+1) ;
$$

time $(t)$ changes by one unit after each trading. The steady-state $(t \rightarrow \infty)$ distribution of money is Gibbs one:

$$
P(m)=(1 / T) \exp (-m / T) ; T=M / N .
$$

Hence, no matter how uniform or justified the initial distribution is, the eventual steady state corresponds to Gibbs distribution where most of the 
people have got very little money. This follows from the conservation of money and additivity of entropy:

$$
P\left(m_{1}\right) P\left(m_{2}\right)=P\left(m_{1}+m_{2}\right) .
$$

This steady state result is quite robust and realistic too! In fact, several variations of the trading, and of the 'lattice' (on which the agents can be put and each agent trade with its 'lattice neighbors' only), whether compact, fractal or small-world like [2], leaves the distribution unchanged. Some other variations like random sharing of an amount $2 m_{2}$ only (not of $m_{1}+m_{2}$ ) when $m_{1}>m_{2}$ (trading at the level of lower economic class in the trade), lead to even drastic situation: all the money in the market drifts to one agent and the rest become truely pauper [9, 10].

\subsection{Effect of fixed or uniform savings}

In any trading, savings come naturally [18]. A saving propensity factor $\lambda$ is therefore introduced in the same model $[\underline{8}$ (see [7] for model without savings), where each trader at time $t$ saves a fraction $\lambda$ of its money $m_{i}(t)$ and trades randomly with the rest:

$$
m_{i}(t+1)=m_{i}(t)+\Delta m ; \quad m_{j}(t+1)=m_{j}(t)-\Delta m
$$

where

$$
\Delta m=(1-\lambda)\left[\epsilon\left\{m_{i}(t)+m_{j}(t)\right\}-m_{i}(t)\right]
$$

$\epsilon$ being a random fraction, coming from the stochastic nature of the trading.
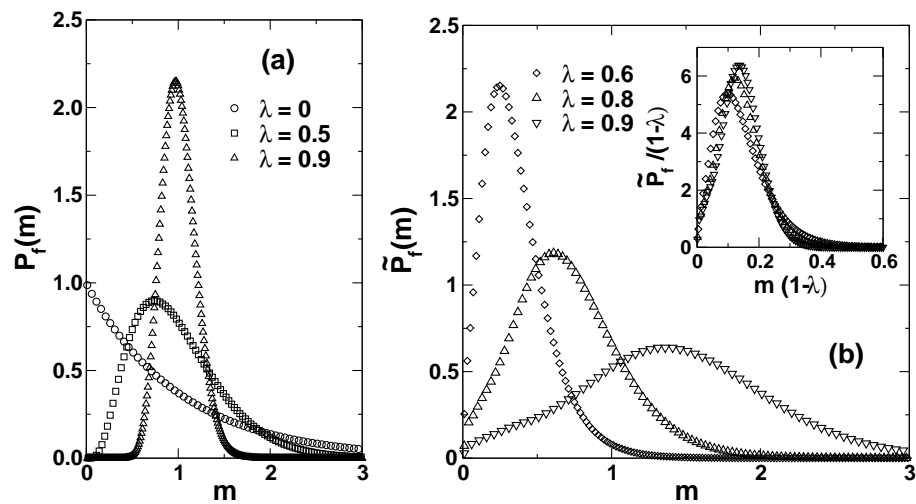

Fig. 1. Steady state money distribution (a) $P(m)$ for the fixed $\lambda$ model, and (b) $\tilde{P}_{f}(m)$ for some specific values of $\lambda$ in the distributed $\lambda$ model. All data are for $N=200$. Inset of (b) shows scaling behavior of $\tilde{P}_{f}(m)$. 
The market (non-interacting at $\lambda=0$ and 1) becomes 'interacting' for any non-vanishing $\lambda(<1)$ : For fixed $\lambda$ (same for all agents), the steady state distribution $P_{f}(m)$ of money is exponentially decaying on both sides with the most-probable money per agent shifting away from $m=0$ (for $\lambda=0$ ) to $M / N$ as $\lambda \rightarrow 1$ (Fig. 1(a)). This self-organizing feature of the market, induced by sheer self-interest of saving by each agent without any global perspective, is quite significant as the fraction of paupers decrease with saving fraction $\lambda$ and most people end up with some fraction of the average money in the market (for $\lambda \rightarrow 1$, the socialists' dream is achieved with just people's self-interest of saving!). Interestingly, self-organisation also occurs in such market models when there is restriction in the commodity market [11. Although this fixed saving propensity does not give yet the Pareto-like power-law distribution, the Markovian nature of the scattering or trading processes (eqn. (4)) is lost and the system becomes co-operative. Indirectly through $\lambda$, the agents get to know (start interacting with) each other and the system co-operatively self-organises towards a most-probable distribution $\left(m_{p} \neq 0\right)$.

This has been understood to a certain extent (see e.g, [20, 21]), and argued to resemble a gamma distribution [21], and partly explained analytically [22].

\subsection{Effect of distributed savings}

In a real society or economy, $\lambda$ is a very inhomogeneous parameter: the interest of saving varies from person to person. We move a step closer to the real situation where saving factor $\lambda$ is widely distributed within the population 12, 13, 14.

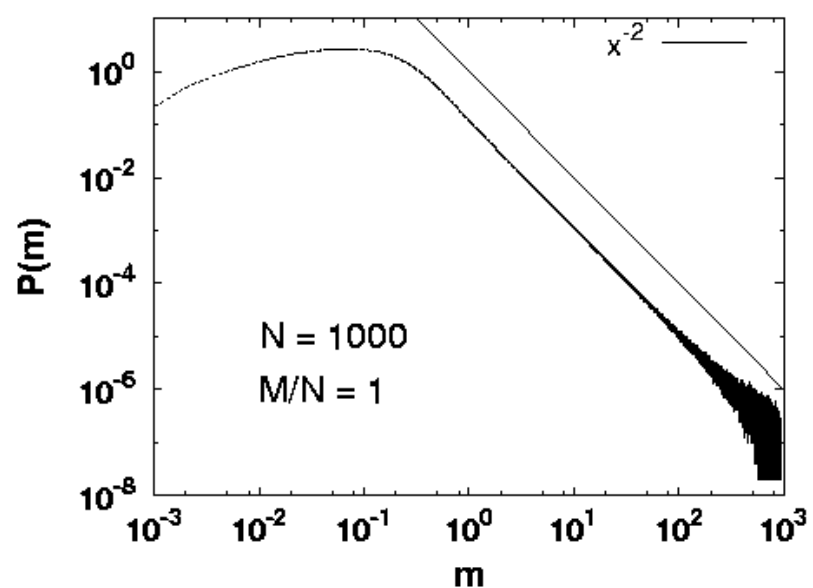

Fig. 2. Steady state money distribution $P(m)$ for the distributed $\lambda$ model with $0 \leq \lambda<1$ for a system of $N=1000$ agents. The $x^{-2}$ is a guide to the observed power-law, with $1+\nu=2$. 
The evolution of money in such a trading can be written as:

$$
\begin{gathered}
m_{i}(t+1)=\lambda_{i} m_{i}(t)+\epsilon_{i j}\left[\left(1-\lambda_{i}\right) m_{i}(t)+\left(1-\lambda_{j}\right) m_{j}(t)\right], \\
m_{j}(t+1)=\lambda_{j} m_{j}(t)+\left(1-\epsilon_{i j}\right)\left[\left(1-\lambda_{i}\right) m_{i}(t)+\left(1-\lambda_{j}\right) m_{j}(t)\right]
\end{gathered}
$$

One again follows the same trading rules as before, except that

$$
\Delta m=\epsilon_{i j}\left(1-\lambda_{j}\right) m_{j}(t)-\left(1-\lambda_{i}\right)\left(1-\epsilon_{i j}\right) m_{i}(t)
$$

here; $\lambda_{i}$ and $\lambda_{j}$ being the saving propensities of agents $i$ and $j$. The agents have fixed (over time) saving propensities, distributed independently, randomly and uniformly (white) within an interval 0 to 1 agent $i$ saves a random fraction $\lambda_{i}\left(0 \leq \lambda_{i}<1\right)$ and this $\lambda_{i}$ value is quenched for each agent $\left(\lambda_{i}\right.$ are independent of trading or $t$ ). Starting with an arbitrary initial (uniform or random) distribution of money among the agents, the market evolves with the tradings. At each time, two agents are randomly selected and the money exchange among them occurs, following the above mentioned scheme. We check for the steady state, by looking at the stability of the money distribution in successive Monte Carlo steps $t$ (we define one Monte Carlo time step as $N$ pairwise interations). Eventually, after a typical relaxation time $\left(\sim 10^{6}\right.$ for $N=1000$ and uniformly distributed $\lambda$ ) dependent on $N$ and the distribution of $\lambda$, the money distribution becomes stationary. After this, we average the money distribution over $\sim 10^{3}$ time steps. Finally we take configurational average over $\sim 10^{5}$ realizations of the $\lambda$ distribution to get the money distribution $P(m)$. It is found to follow a strict power-law decay. This decay fits to Pareto law (11) with $\nu=1.01 \pm 0.02$ (Fig. 2). Note, for finite size $N$ of the market, the distribution has a narrow initial growth upto a most-probable value $m_{p}$ after which it falls off with a power-law tail for several decades. This Pareto law (with $\nu \simeq 1$ ) covers the entire range in $m$ of the distribution $P(m)$ in the limit $N \rightarrow \infty$. We checked that this power law is extremely robust: apart from the uniform $\lambda$ distribution used in the simulations in Fig. 2, we also checked the results for a distribution

$$
\rho(\lambda) \sim\left|\lambda_{0}-\lambda\right|^{\alpha}, \quad \lambda_{0} \neq 1, \quad 0<\lambda<1,
$$

of quenched $\lambda$ values among the agents. The Pareto law with $\nu=1$ is universal for all $\alpha$. The data in Fig. 2 corresponds to $\lambda_{0}=0, \alpha=0$. For negative $\alpha$ values, however, we get an initial (small $m$ ) Gibbs-like decay in $P(m)$ (see Fig. 3).

In case of uniform distribution of saving propensity $\lambda(0 \leq \lambda<1)$, the individual money distribution $\tilde{P}_{f}(m)$ for agents with any particular $\lambda$ value, although differs considerably, remains non-monotonic: similar to that for fixed $\lambda$ market with $m_{p}(\lambda)$ shifting with $\lambda$ (see Fig. 1). Few subtle points may be noted though: while for fixed $\lambda$ the $m_{p}(\lambda)$ were all less than of the order of unity (Fig. 1(a)), for distributed $\lambda$ case $m_{p}(\lambda)$ can be considerably larger and can approach to the order of $N$ for large $\lambda$ (see Fig. 1(b)). The other important 


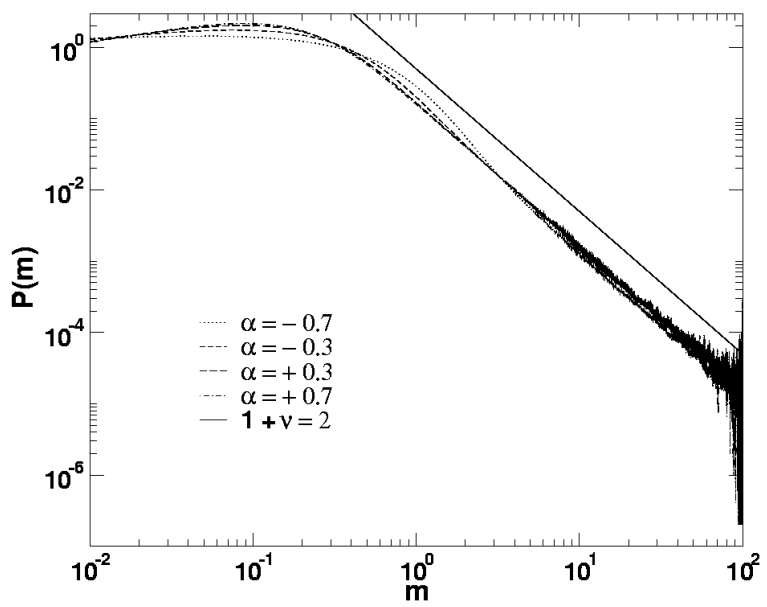

Fig. 3. Steady state money distribution $P(m)$ in the model with for a system of $N=100$ agents with $\lambda$ distributed as $\rho(\lambda) \sim \lambda^{\alpha}$, with different values of $\alpha$. In all cases, agents play with average money per agent $M / N=1$.

difference is in the scaling behavior of $\tilde{P}_{f}(m)$, as shown in the inset of Fig. 1(b). In the distributed $\lambda$ ensemble, $\tilde{P}_{f}(m)$ appears to have a very simple scaling:

$$
\tilde{P}_{f}(m) \sim(1-\lambda) \mathcal{F}(m(1-\lambda)),
$$

for $\lambda \rightarrow 1$, where the scaling function $\mathcal{F}(x)$ has non-monotonic variation in $x$. The fixed (same for all agents) $\lambda$ income distribution $P_{f}(m)$ do not have any such comparative scaling property. It may be noted that a small difference exists between the ensembles considered in Fig 1(a) and 1(b): while $\int m P_{f}(m) d m=M$ (independent of $\lambda$ ), $\int m \tilde{P}_{f}(m) d m$ is not a constant and infact approaches to order of $M$ as $\lambda \rightarrow 1$. There is also a marked qualitative difference in fluctuations (see Fig. 4): while for fixed $\lambda$, the fluctuations in time (around the most-probable value) in the individuals' money $m_{i}(t)$ gradually decreases with increasing $\lambda$, for quenched distribution of $\lambda$, the trend gets reversed (see Fig. 4).

We now investigate on the range of distribution of the saving propensities in a certain interval $a<\lambda_{i}<b$, where, $0<a<b<1$. For uniform distribution within the range, we observe the appearance of the same power law in the distribution but for a narrower region. As may be seen from Fig. 5, as $a \rightarrow b$, the power-law behavior is seen for values $a$ or $b$ approaching more and more towards unity: For the same width of the interval $|b-a|$, one gets power-law (with same $\nu$ ) when $b \rightarrow 1$. This indicates, for fixed $\lambda, \lambda=0$ corresponds to Gibbs distribution, and one gets Pareto law when $\lambda$ has got non-zero width of its distribution extending upto $\lambda=1$. This of course indicates a crucial role of these high saving propensity agents: the power law behavior is truely valid upto the asymptotic limit if $\lambda=1$ is included. Indeed, had we assumed $\lambda_{0}=1$ 

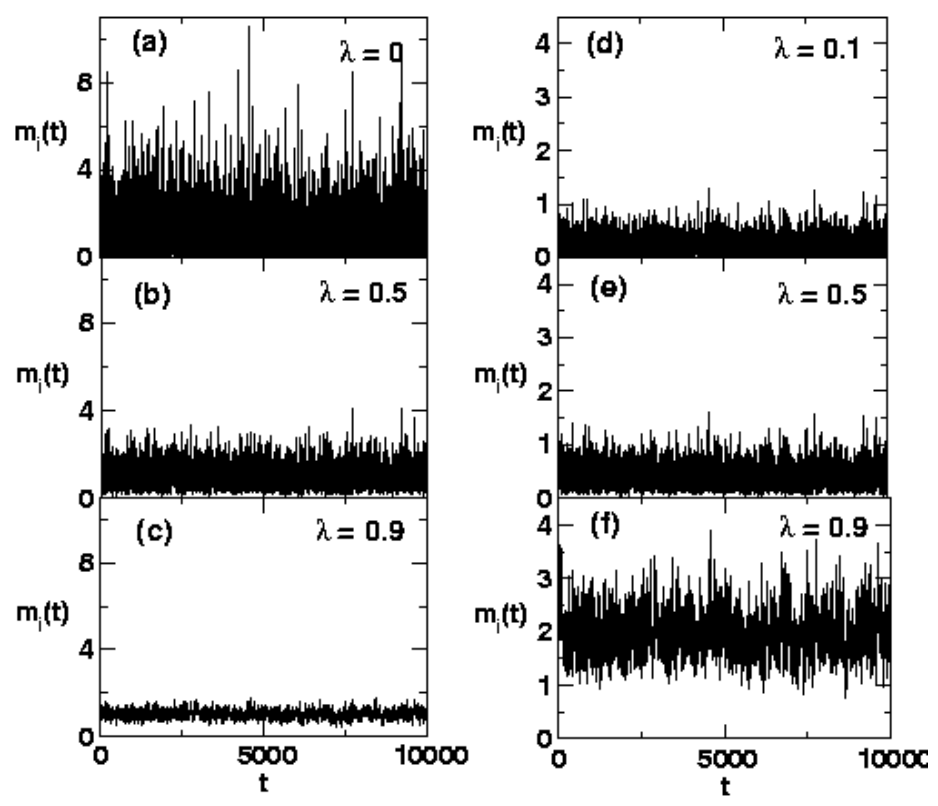

Fig. 4. Time variation of the money of the $i$ th trader: For fixed $\lambda$ market $-(a)$, (b), (c); and for agents with specific values of $\lambda$ in the distributed $\lambda$ market $-(d)$, (e), (f).

in (10), the Pareto exponent $\nu$ immediately switches over to $\nu=1+\alpha$. Of course, $\lambda_{0} \neq 1$ in (10) leads to the universality of the Pareto distribution with $\nu=1$ (irrespective of $\lambda_{0}$ and $\alpha$ ). Indeed this can be easily rationalised from the scaling behavior (111): $P(m) \sim \int_{0}^{1} \tilde{P}_{f}(m) \rho(\lambda) d \lambda \sim m^{-2}$ for $\rho(\lambda)$ given by (10) and $m^{-(2+\alpha)}$ if $\lambda_{0}=1$ in (10) (for large $m$ values).

These model income distributions $P(m)$ compare very well with the wealth distributions of various countries: Data suggests Gibbs like distribution in the low-income range (more than $90 \%$ of the population) and Pareto-like in the high-income range [3] (less than $10 \%$ of the population) of various countries. In fact, we compared one model simulation of the market with saving propensity of the agents distributed following (10), with $\lambda_{0}=0$ and $\alpha=-0.7$ [12]. The qualitative resemblance of the model income distribution with the real data for Japan and USA in recent years is quite intriguing. In fact, for negative $\alpha$ values in (10), the density of traders with low saving propensity is higher and since $\lambda=0$ ensemble yields Gibbs-like income distribution (3), we see an initial Gibbs-like distribution which crosses over to Pareto distribution (10) with $\nu=1.0$ for large $m$ values. The position of the crossover point depends on the value of $\alpha$. It is important to note that any distribution of $\lambda$ near $\lambda=1$, of finite width, eventually gives Pareto law for large $m$ limit. The same 


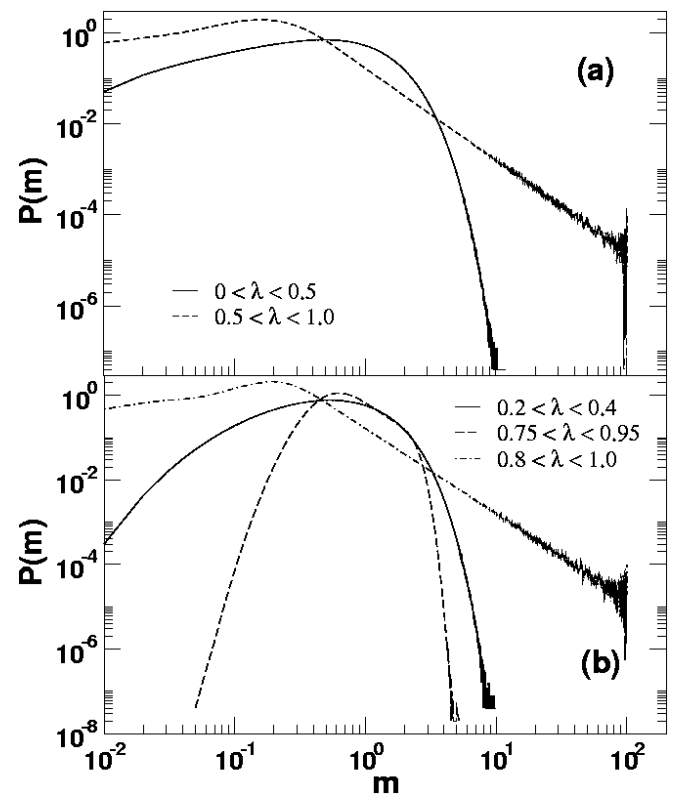

Fig. 5. Steady state money distribution in cases when the saving propensity $\lambda$ is distributed uniformly within a range of values: (a) width of $\lambda$ distribution is 0.5 , money distribution shows power law for $0.5<\lambda<1.0$; (a) width of $\lambda$ distribution is 0.2 , money distribution shows power law for $0.7<\lambda<0.9$. The power law exponent is $\nu \simeq 1$ in all cases. All data shown here are for $N=100, M / N=1$.

kind of crossover behavior (from Gibbs to Pareto) can also be reproduced in a model market of mixed agents where $\lambda=0$ for a finite fraction of population and $\lambda$ is distributed uniformly over a finite range near $\lambda=1$ for the rest of the population.

We even considered annealed randomness in the saving propensity $\lambda$ : here $\lambda_{i}$ for any agent $i$ changes from one value to another within the range $0 \leq$ $\lambda_{i}<1$, after each trading. Numerical studies for this annealed model did not show any power law behavior for $P(m)$; rather it again becomes exponentially decaying on both sides of a most-probable value.

\section{Dynamics of money exchange}

We will now investigate the steady state distribution of money resulting from the above two equations representing the trading and money dynamics. We will now solve the dynamics of money distribution in two limits. In one case, we study the evolution of the mutual money difference among the agents and 
look for a self-consistent equation for its steady state distribution. In the other case, we develop a master equation for the money distribution function.

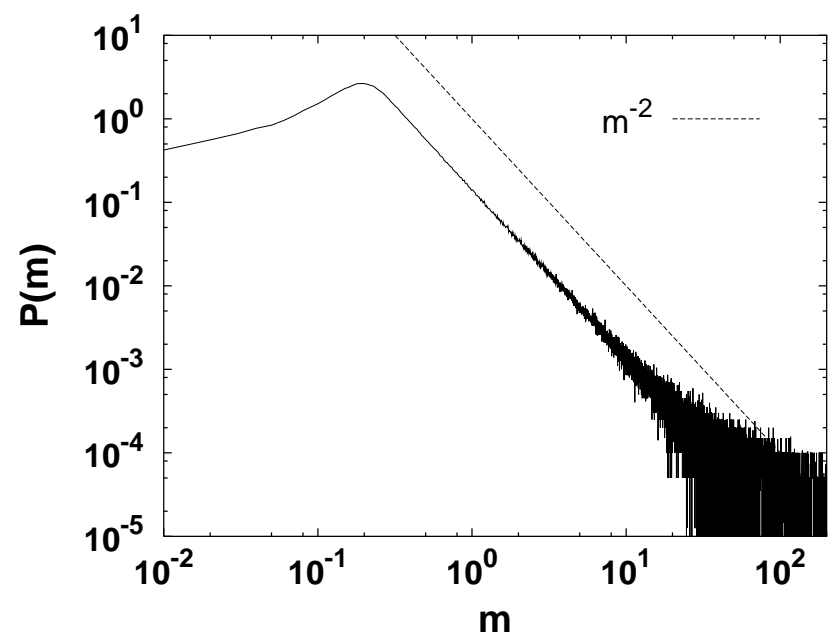

Fig. 6. Steady state money distribution $P(m)$ against $m$ in a numerical simulation of a market with $N=200$, following equations (7) and (8) with $\epsilon_{i j}=1 / 2$. The dotted lines correspond to $m^{-(1+\nu)} ; \nu=1$.

\subsection{Distribution of money difference}

Clearly in the process as considered above, the total money $\left(m_{i}+m_{j}\right)$ of the pair of agents $i$ and $j$ remains constant, while the difference $\Delta m_{i j}$ evolves as

$$
\begin{aligned}
\left(\Delta m_{i j}\right)_{t+1} \equiv & \left(m_{i}-m_{j}\right)_{t+1} \\
= & \left(\frac{\lambda_{i}+\lambda_{j}}{2}\right)\left(\Delta m_{i j}\right)_{t}+\left(\frac{\lambda_{i}-\lambda_{j}}{2}\right)\left(m_{i}+m_{j}\right)_{t} \\
& +\left(2 \epsilon_{i j}-1\right)\left[\left(1-\lambda_{i}\right) m_{i}(t)+\left(1-\lambda_{j}\right) m_{j}(t)\right] .
\end{aligned}
$$

Numerically, as shown in Fig. 2, we observe that the steady state money distribution in the market becomes a power law, following such tradings when the saving factor $\lambda_{i}$ of the agents remain constant over time but varies from agent to agent widely. As shown in the numerical simulation results for $P(m)$ in Fig. 6, the law, as well as the exponent, remains unchanged even when $\epsilon_{i j}=1 / 2$ for every trading. This can be justified by the earlier numerical observation [8, 12 for fixed $\lambda$ market $\left(\lambda_{i}=\lambda\right.$ for all $\left.i\right)$ that in the steady state, criticality occurs as $\lambda \rightarrow 1$ where of course the dynamics becomes extremely slow. In other words, after the steady state is realized, the third term in (12) becomes unimportant for the critical behavior. We therefore concentrate on 
this case, where the above evolution equation for $\Delta m_{i j}$ can be written in a more simplified form as

$$
\left(\Delta m_{i j}\right)_{t+1}=\alpha_{i j}\left(\Delta m_{i j}\right)_{t}+\beta_{i j}\left(m_{i}+m_{j}\right)_{t}
$$

where $\alpha_{i j}=\frac{1}{2}\left(\lambda_{i}+\lambda_{j}\right)$ and $\beta_{i j}=\frac{1}{2}\left(\lambda_{i}-\lambda_{j}\right)$. As such, $0 \leq \alpha<1$ and $-\frac{1}{2}<\beta<\frac{1}{2}$.

The steady state probability distribution $D$ for the modulus $\Delta=|\Delta m|$ of the mutual money difference between any two agents in the market can be obtained from (13) in the following way provided $\Delta$ is very much larger than the average money per agent $=M / N$. This is because, large $\Delta$ can appear from 'scattering' involving $m_{i}-m_{j}= \pm \Delta$ and when either $m_{i}$ or $m_{j}$ is small. When both $m_{i}$ and $m_{j}$ are large, maintaining a large $\Delta$ between them, their probability is much smaller and hence their contribution. Then if, say, $m_{i}$ is large and $m_{j}$ is not, the right hand side of (13) becomes $\sim\left(\alpha_{i j}+\beta_{i j}\right)\left(\Delta_{i j}\right)_{t}$ and so on. Consequently for large $\Delta$ the distribution $D$ satisfies

$$
\begin{aligned}
D(\Delta) & =\int d \Delta^{\prime} D\left(\Delta^{\prime}\right)\left\langle\delta\left(\Delta-(\alpha+\beta) \Delta^{\prime}\right)+\delta\left(\Delta-(\alpha-\beta) \Delta^{\prime}\right)\right\rangle \\
& =2\left\langle\left(\frac{1}{\lambda}\right) D\left(\frac{\Delta}{\lambda}\right)\right\rangle,
\end{aligned}
$$

where we have used the symmetry of the $\beta$ distribution and the relation $\alpha_{i j}+\beta_{i j}=\lambda_{i}$, and have suppressed labels $i, j$. Here $\langle\ldots\rangle$ denote average over $\lambda$ distribution in the market. Taking now a uniform random distribution of the saving factor $\lambda, \rho(\lambda)=1$ for $0 \leq \lambda<1$, and assuming $D(\Delta) \sim \Delta^{-(1+\gamma)}$ for large $\Delta$, we get

$$
1=2 \int d \lambda \lambda^{\gamma}=2(1+\gamma)^{-1}
$$

giving $\gamma=1$. No other value fits the above equation. This also indicates that the money distribution $P(m)$ in the market also follows a similar power law variation, $P(m) \sim m^{-(1+\nu)}$ and $\nu=\gamma$. We will now show in a more rigorous way that indeed the only stable solution corresponds to $\nu=1$, as observed numerically [12, 13, 14].

\subsection{Master equation and its analysis}

We also develop a Boltzmann-like master equation for the time development of $P(m, t)$, the probability distribution of money in the market [25, 26]. We again consider the case $\epsilon_{i j}=\frac{1}{2}$ in (7) and (8) and rewrite them as

$$
\left(\begin{array}{c}
m_{i} \\
m_{j}
\end{array}\right)_{t+1}=\mathcal{A}\left(\begin{array}{c}
m_{i} \\
m_{j}
\end{array}\right)_{t} \text { where } \mathcal{A}=\left(\begin{array}{cc}
\mu_{i}^{+} & \mu_{j}^{-} \\
\mu_{i}^{-} & \mu_{j}^{+}
\end{array}\right) ; \quad \mu^{ \pm}=\frac{1}{2}(1 \pm \lambda) .
$$

Collecting the contributions from terms scattering in and subtracting those scattering out, we can write the master equation for $P(m, t)$ as 
$\frac{\partial P(m, t)}{\partial t}+P(m, t)=\left\langle\int d m_{i} \int d m_{j} P\left(m_{i}, t\right) P\left(m_{j}, t\right) \delta\left(\mu_{i}^{+} m_{i}+\mu_{j}^{-} m_{j}-m\right)\right\rangle$,

which in the steady state gives

$$
P(m)=\left\langle\int d m_{i} \int d m_{j} P\left(m_{i}\right) P\left(m_{j}\right) \delta\left(\mu_{i}^{+} m_{i}+\mu_{j}^{-} m_{j}-m\right)\right\rangle .
$$

Assuming, $P(m) \sim m^{-(1+\nu)}$ for $m \rightarrow \infty$, we get [25, 26]

$$
1=\left\langle\left(\mu^{+}\right)^{\nu}+\left(\mu^{-}\right)^{\nu}\right\rangle \equiv \iint d \mu^{+} d \mu^{-} p\left(\mu^{+}\right) q\left(\mu^{-}\right)\left[\left(\mu^{+}\right)^{\nu}+\left(\mu^{-}\right)^{\nu}\right] .
$$

Considering now the dominant terms $\left(\propto x^{-r}\right.$ for $r>0$, or $\propto \ln (1 / x)$ for $\left.r=0\right)$ in the $x \rightarrow 0$ limit of the integral $\int_{0}^{\infty} m^{(\nu+r)} P(m) \exp (-m x) d m$, we get from eqn. (19), after integrations, $1=2 /(\nu+1)$, giving finally $\nu=1$ (details in Appendix).

\section{Summary and Discussions}

We have numerically simulated here ideal-gas like models of trading markets, where each agent is identified with a gas molecule and each trading as an elastic or money-conserving two-body collision. Unlike in the ideal gas, we introduce (quenched) saving propensity of the agents, distributed widely between the agents $(0 \leq \lambda<1)$. For quenched random variation of $\lambda$ among the agents the system remarkably self-organizes to a critical Pareto distribution (11) of money with $\nu \simeq 1.0$ (Fig. 2). The exponent is quite robust: for savings distribution $\rho(\lambda) \sim\left|\lambda_{0}-\lambda\right|^{\alpha}, \lambda_{0} \neq 1$, one gets the same Pareto law with $\nu=1$ (independent of $\lambda_{0}$ or $\alpha$ ).

A master equation for $P(m, t)$, as in (17), for the original case (eqns. (7) and (8) $)$ was first formulated for fixed $\lambda\left(\lambda_{i}\right.$ same for all $\left.i\right)$, in [20] and solved numerically. Later, a generalized master equation for the same, where $\lambda$ is distributed, was formulated and solved in 22 and 25. We show here that our analytic study clearly support the power-law for $P(m)$ with the exponent value $\nu=1$ universally, as observed numerically earlier [12, 13, 14.

It may be noted that the trading market model we have talked about here has got some apparent limitations. The stochastic nature of trading assumed here in the trading market, through the random fraction $\epsilon$ in ([6), is of course not very straightforward as agents apparently go for trading with some definite purpose (utility maximization of both money and commodity). We are however, looking only at the money transactions between the traders. In this sense, the income distribution we study here essentially corresponds to 'paper money', and not the 'real wealth'. However, even taking money and commodity together, one can argue (see [10]) for the same stochastic nature of the tradings, due to the absence of 'just pricing' and the effects of bargains in the market. 
Apart from the observation that Gibbs (1901) and Pareto (1897) distributions fall in the same category and can appear naturally in the century-old and well-established kinetic theory of gas, that this model study indicates the appearance of self-organized criticality in the simplest (gas) model so far, when the stability effect of savings incorporated, is remarkable.

\section{Acknowledgments}

We are grateful to A. Chakraborti, S. Pradhan, S. S. Manna and R. B. Stinchcombe for collaborations at various stages of our study.

\section{A Alternative solution of the steady state master equation (18)}

Let $S_{r}(x)=\int_{0}^{\infty} d m P(m) m^{\nu+r} \exp (-m x) ; r \geq 0, x>0$. If $P(m)=A / m^{1+\nu}$, then

$$
\begin{aligned}
S_{r}(x) & =A \int_{0}^{\infty} d m m^{r-1} \exp (-m x) \\
& \sim A \frac{x^{-r}}{r} \quad \text { if } r>0 \\
& \sim A \ln \left(\frac{1}{x}\right) \quad \text { if } r=0 .
\end{aligned}
$$

From eqn. (18), we can write

$$
\begin{aligned}
& S_{r}(x)= \\
& \left\langle\int_{0}^{\infty} d m_{i} \int_{0}^{\infty} d m_{j} P\left(m_{i}\right) P\left(m_{j}\right)\left(m_{i} \mu_{i}^{+}+m_{j} \mu_{j}^{-}\right)^{\nu+r} \exp \left[-\left(m_{i} \mu_{i}^{+}+m_{j} \mu_{j}^{-}\right) x\right]\right\rangle \\
\simeq & \int_{0}^{\infty} d m_{i} A m_{i}^{r-1}\left\langle\exp \left(-m_{i} \mu_{i}^{+} x\right)\left(\mu_{i}^{+}\right)^{\nu+r}\right\rangle\left[\int_{0}^{\infty} d m_{j} P\left(m_{j}\right)\left\langle\exp \left(-m_{j} \mu_{j}^{-} x\right)\right\rangle\right] \\
+ & \int_{0}^{\infty} d m_{j} A m_{j}^{r-1}\left\langle\exp \left(-m_{j} \mu_{j}^{-} x\right)\left(\mu_{j}^{-}\right)^{\nu+r}\right\rangle\left[\int_{0}^{\infty} d m_{i} P\left(m_{i}\right)\left\langle\exp \left(-m_{i} \mu_{i}^{+} x\right)\right\rangle\right]
\end{aligned}
$$

or,

$$
\begin{aligned}
S_{r}(x)= & \int_{\frac{1}{2}}^{1} d \mu_{i}^{+} p\left(\mu_{i}^{+}\right)\left(\int_{0}^{\infty} d m_{i} A m_{i}^{r-1} \exp \left(-m_{i} \mu_{i}^{+} x\right)\right)\left(\mu_{i}^{+}\right)^{\nu+r} \\
& +\int_{0}^{\frac{1}{2}} d \mu_{j}^{-} q\left(\mu_{j}^{-}\right)\left(\int_{0}^{\infty} d m_{j} A m_{j}^{r-1} \exp \left(-m_{j} \mu_{j}^{-} x\right)\right)\left(\mu_{j}^{-}\right)^{\nu+r}
\end{aligned}
$$


since for small $x$, the terms in the square brackets in (21) approach unity. We can therefore rewrite (22) as

$$
S_{r}(x)=2\left[\int_{\frac{1}{2}}^{1} d \mu^{+}\left(\mu^{+}\right)^{\nu+r} S_{r}\left(x \mu^{+}\right)+\int_{0}^{\frac{1}{2}} d \mu^{-}\left(\mu^{-}\right)^{\nu+r} S_{r}\left(x \mu^{-}\right)\right] .
$$

Using now the forms of $S_{r}(x)$ as in (20), and collecting terms of order $x^{-r}$ (for $r>0$ ) or of order $\ln (1 / x)$ (for $r=0$ ) from both sides of (23), we get (19).

\section{References}

1. Pareto V (1897) Cours d'economie Politique. F. Rouge, Lausanne

2. Moss de Oliveira S, de Oliveira PMC, Stauffer D (1999) Evolution, Money, War and Computers. B. G. Tuebner, Stuttgart, Leipzig

3. Levy M, Solomon S (1997) New evidence for the power-law distribution of wealth, Physica A 242:90-94; Drăgulescu AA, Yakovenko VM (2001) Exponential and Power-Law Probability Distributions of Wealth and Income in the United Kingdom and the United States. Physica A 299:213-221; Aoyama H, Souma W, Fujiwara Y (2003) Growth and fluctuations of personal and company's income, Physica A 324:352

4. Di Matteo T, Aste T, Hyde ST (2003) Exchanges in Complex Networks: Income and Wealth Distributions, cond-mat/0310544 Clementi F, Gallegati M (2005), Power Law Tails in the Italian Personal Income Distribution. Physica A $350: 427-438$

5. Sinha S (2005) Evidence for Power-law Tail of the Wealth Distribution in India, cond-mat/0502166

6. Chakrabarti BK, Marjit S (1995) Self-organization in Game of Life and Economics,Indian J. Phys. B69:681-698; Ispolatov S, Krapivsky PL, Redner S (1998) Wealth distributions in asset exchange models, Eur. Phys. J. B 2:267;

7. Drăgulescu AA, Yakovenko VM (2000) Statistical Mechanics of Money, Eur. Phys. J. B 17:723-726

8. Chakraborti A, Chakrabarti BK (2000) Statistical Mechanics of Money: Effects of Saving Propensity, Eur. Phys. J. B 17:167-170

9. Chakraborti A (2002) Distributions of money in model markets of economy, Int. J. Mod. Phys. C 13:1315

10. Hayes B (2002) Follow the Money, American Scientist, USA, 90:(Sept-Oct)400405

11. Chakraborti A, Pradhan S, Chakrabarti BK (2001) A Self-organizing Market Model with single Commodity, Physica A 297:253-259

12. Chatterjee A, Chakrabarti BK, Manna SS (2004) Pareto Law in a Kinetic Model of Market with Random Saving Propensity, Physica A 335:155

13. Chatterjee A, Chakrabarti BK; Manna SS (2003) Money in Gas-like Markets: Gibbs and Pareto Laws, Physica Scripta T 106:36

14. Chakrabarti BK, Chatterjee A (2004) Ideal Gas-Like Distributions in Economics: Effects of Saving Propensity, in Application of Econophysics, Proc. 2nd Nikkei Econophys. Symp., Ed. Takayasu H, Springer, Tokyo, pp. 280-285 
15. Sinha S (2003) Stochastic Maps, Wealth Distribution in Random Asset Exchange Models and the Marginal Utility of Relative Wealth, Phys. Scripta T106:59-64; Ferrero JC (2004) The statistical distribution of money and the rate of money transference, Physica A 341:575; Iglesias JR, Gonçalves S, Abramson G, Vega JL (2004) Correlation between risk aversion and wealth distribution, Physica A 342:186; Scafetta N, Picozzi S, West BJ (2004) A trade-investment model for distribution of wealth, Physica D 193:338-352

16. Slanina F (2004) Inelastically scattering particles and wealth distribution in an open economy, Phys. Rev. E 69:046102

17. See e.g, Landau LD, Lifshitz EM (1968), Statistical Physics. Pergamon Press, Oxford.

18. Samuelson PA (1980) Economics. Mc-Graw Hill Int., Auckland.

19. Willis G, Mimkes J (2004) Evidence for the Independence of Waged and Unwaged Income, Evidence for Boltzmann Distributions in Waged Income, and the Outlines of a Coherent Theory of Income Distribution, cond-mat/0406694

20. Das A, Yarlagadda S (2003) Analytic treatment of a trading market model, Phys. Scripta T106:39-40

21. Patriarca M, Chakraborti A, Kaski K (2004) A Statistical model with a standard $\Gamma$ distribution, Phys. Rev. E 70:016104

22. Repetowicz P, Hutzler S, Richmond P (2004) Dynamics of Money and Income Distributions, cond-mat/0407770

23. Ding N, Xi N, Wang Y (2003) Effects of saving and spending patterns on holding time distribution, Eur. Phys. J. B 36:149

24. Bak P (1997) How Nature works. Oxford University Press, Oxford.

25. Chatterjee A, Chakrabarti BK, Stinchcombe RB (2005) Master equation for a kinetic model of trading market and its analytic solution, cond-mat/0501413

26. Chatterjee A, Chakrabarti BK, Stinchcombe RB (2005) Analyzing money distributions in 'ideal gas' models of markets, in 'Practical Fruits of Econophysics', Ed. Takayasu H, Springer-Berlag, Tokyo Proc. Third Nikkei Symposium on Econophysics, Tokyo, Japan, 2004, cond-mat/0501413

27. Dynan KE, Skinner J, Zeldes SP (2004) Do the rich save more ?, J. Pol. Econ. 112: 397-444. 